\title{
Lowering the female hairline: two options in treatment
}

\author{
Jeffrey Epstein, MD, FACS, Ashlin Alexander, MD New York, New York, USA jsemd@fhrps.com
}

\section{Introduction}

One of our main roles as hair restoration surgeons, whether we recognize it every day, is to achieve a balance between the various parts of the face. The guiding principle of equal vertical thirds dictates that the height of the forehead (the distance from the glabella to the trichion) should compose approximately one-third the vertical height of the face. In women in particular, a lower anterior hairline, and thus shorter forehead height, are considered more attractive and youthful. A longer forehead, meanwhile, can be viewed as unaesthetic, somewhat masculine, and connote senescence.

To lower the female hairline, most hair transplant surgeons will utilize hair grafting, and for good reason, which will be discussed below. However, there is a definite group of female patients that is much better treated by and will achieve more aesthetic and impressive results from surgical hairline advancement (SHA), a procedure that I learned from Dr. Sheldon Kabaker. Over the past three years, SHA has become the senior author's favorite surgery to perform, due to the dramatic results and tremendously high rate of satisfaction for both the patient and the surgeon (Figures 1-3), and is thus reserved for that small but definite $15 \%-20 \%$ of women who are the best candidates.

Before going into the surgical details of each of these two procedures, a review of the comparative indications is provided.

\section{Patient Evaluation and Proper Indications for Each Procedure}

A high hairline in a woman can be of iatrogenic etiology (prior brow lifting, traction alopecia), present at birth, or due to frontal hair loss most commonly from androgenic female pattern balding. Pre-operatively, it is critical to determine whether the already high hairline is stable (as a result of genetics or prior brow lifting) or progressive. If progressive, factors to consider include the rate of the hair loss, treatments that have been employed and their efficacy, and possible etiologies including certain hair styles such as tight braids that could cause traction alopecia, or medical conditions such

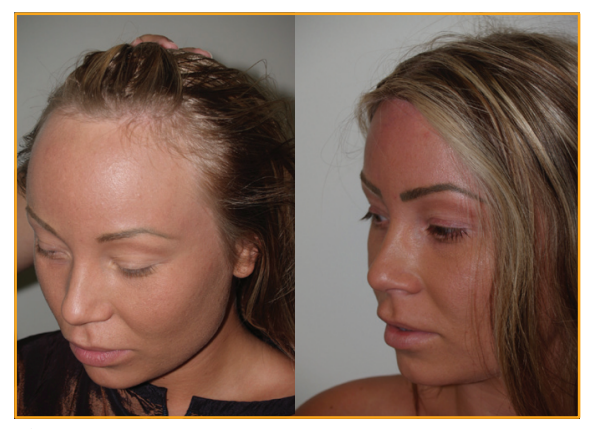

Figure 3. Before (left) and 2 years after SHA (right), performed as a single procedure. (This procedure is available for viewing.)

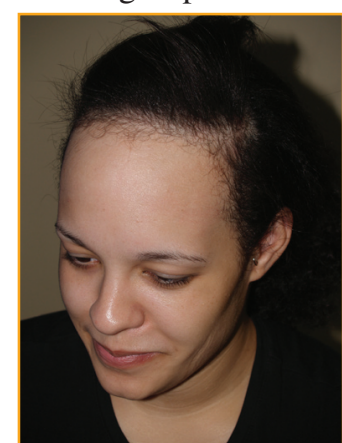

Figure 1. Before surgical hairline advancement.

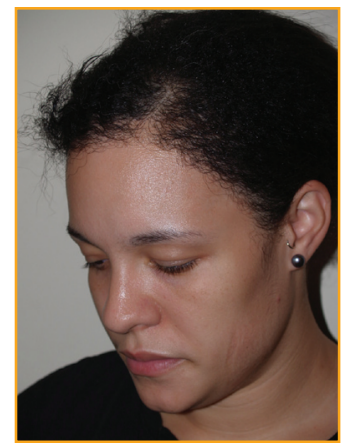

Figure 2. Patient 1 year after surgical hairline advancement and 7 months after hair grafting. She first underwent SHA to advance the hairline, then 5 months late had a transplant of 650 grafts to round out and soften the hairline. stable hairline are good candidates for SHA, otherwise future hair loss could expose the hairline incision while reversing the achievement of a lower hairline, and also the performing of the procedure could cause further hair loss (telogen effluvium).

A pertinent physical exam includes an assessment of skin type and laxity, symmetry of the hairline, and the presence of any brow ptosis (that can optionally be addressed with SHA). The laxity of the scalp is critical for determining how much the hairline can be surgically advanced and thus achieve the patient's goals. Laxity assessment is discussed in detail below.

The pattern of hair loss (including miniaturization of hairs along the hairline, implying a risk of shock loss that can result from SHA), the degree of recession of the frontotemporal and temporal hairlines to go along with the high frontal hairline, and the density of hair in the donor area to determine the potential adequacy for hair transplantation, are other elements to be assessed. It is also important to determine the patient's goals, including the position and shape of the hairline, the desire to treat any other possible areas of the scalp that are experiencing hair loss, and whether the patient will be happier with the virtually overnight results with SHA or is willing to wait 6-8 months for transplanted hairs to grow. If rounding out of the hairline-especially into the frontotemporal recession extending into the temporal regions-is desired, because this is not possible with SHA alone, these patients must be advised to undergo either all hair grafting or realize that a hair grafting procedure will need to be performed after SHA in as soon as 3-4 months.

\section{Design of the Hairline}

Simply put, the feminine hairline is straight without temporal receding. Achieving a truly natural appearance requires formulaic planning, artistry, and patient input. Beehner described five key components-height, contour, lateral temporal fringe relationship, natural appearance, and direction and angulation of transplanted hairs-which I use to present a simplified view of how my female hairlines are designed. ${ }^{1}$

The first step is to determine the position of the central-most hairline, typically $6-7 \mathrm{~cm}$ above the glabella, a point that on profile view can be appreciated as correlating with the point where the vertical forehead meets with the horizontal scalp. From this point, where an optional widow's peak can be created, the hairline extends outwards on both sides to create the frontotemporal recessions, which in women can be slightly receded or relatively straight across. Extending further laterally, as opposed to the typical male hairline where there is a relatively sharp transition from the horizontal frontal hairline to the more vertical temporal hairline, women usually have a gentle, curved transition. This temporal hairline proceeds inferiorly in a slightly convex shape, joining up with the temporal tufts/sideburns. I find that a certain percentage of women, especially those of African or Asian ethnicity, desire a more rounded shape to the hairline, where essentially a semi-circle or semi-oval is created from one gentle temporal point to the other.

A review of hair growth direction is covered in the description of surgical technique of hair grafting below. 


\section{Treatment Options: SHA vs. Hair Grafting \\ Surgical Hairline Advancement}

SHA is a one-step procedure that can advance the entire frontal hairline by $1.5 \mathrm{~cm}$ to as much as $5 \mathrm{~cm}$ through a trichophytic (hairline) incision. The advancement of the hairline allows for the corresponding removal of forehead skin, thus shortening the height of the entire forehead. The amount of lowering, the virtually instantaneous results, and the density of the advanced hairline, as well as the ability to aesthetically elevate the eyebrows if desired, is unsurpassed compared to the hair grafting technique, and thus explains the popularity of the procedure for the appropriate and motivated patient.

To be a good candidate, the patient must have adequate scalp mobility to achieve the desired amount of advancement, good frontal hair density, and no history of prior scalp surgery, such as coronal brow lifting, that could compromise healing. A stable frontal hairline is an absolute must, because with progressive hair loss the hairline incision scar could become exposed. In addition, these unstable hairs along the hairline are particularly sensitive to shock loss, thus reversing any gains achieved through the advancement procedure.

Because of how the hairline is advanced in a relatively straightforward fashion, this procedure does not typically permit much rounding out of the hairline; thus, the patient's goal should be achieving a lower, flat hairline. However, the future option of hair grafting as soon as 3 months after SHA is a good one for rounding out the hairline if desired. Additionally, the patient must be willing to accept having a fine, linear scar along the hairline.

To determine scalp laxity, a finger is placed at the centralmost part of the hairline, then moved up and down. The amount of total forward displacement is a relatively accurate predictor of the possible advancement that can be achieved, adding as much as another 5-8mm when galeotomies are performed. Excessive horizontal wrinkling of the skin means another 5-10mm of forehead skin can be excised, adding to the appearance of a lower hairline. Individuals with tight scalps may, if highly motivated, be considered for tissue expansion and subsequent advancement. While very few women are in this category, when performed, tissue expansion can yield tremendous gains in hairline advancement.

Risks of SHA include telogen effluvium, widening of the hairline scar, and potential visibility of the scar with future hair loss. ${ }^{2}$ Patients can also expect decreased frontal scalp sensation for 6-12 months post-operatively.

\section{Surgical Technique}

The fundamental concept of SHA is to physically advance the anterior hairline to a new, lower position. A slightly irregular tricophytic incision line is marked out just along the leading edge of the thicker terminal hairs along the existing frontal hairline. The estimated position of the new hairline, based upon both patient preference and scalp laxity, is marked out along the upper forehead, in a line that runs parallel to the hairline incision. The location of this proposed hairline is determined by a combination of the patient's goals, the surgeon's judgment, and the amount of elasticity of the scalp.

The great majority of these procedures are performed under oral sedation and local anesthesia (with $2 \%$ lidocaine with 1:100,000 epinephrine) blocks of the supraorbital and supratrochlear nerves and infiltration from the forehead back to the vertex, corresponding to the entire scalp region to be undermined. For hemostatic purposes, the two marked lines (trichophytic and estimated new hairline) is similarly infiltrated with a small amount of lidocaine $2 \%$ with 1:100,000 epinephrine. Under sterile conditions, the trichophytic hairline incision is made with a 15scalpel, beveled anterior and cutting $90^{\circ}$ to the exiting hairs so that they are transected so as to promote hair regrowth through the scar. Once deep to the hair follicles, the incision is then made directly downwards, through the galea. Extending laterally, in the temporal regions this incision is made parallel to the hair follicles to prevent injury. These temporal incisions are made within the hair-bearing area approximately $15 \mathrm{~mm}$ posterior to the temporal hairline, and are typically $2-3 \mathrm{~cm}$ long. With the incision made through the galea, wide undermining is then safely and easily performed with a dissecting scissors and/or finger through the essentially avascular subgaleal plane posteriorly and the subfrontalis muscle plane anteriorly. This dissection is carried posteriorly to the vertex, laterally through the superior-most temporal fascia, and anteriorly $1-2 \mathrm{~cm}$ below the anticipated new hairline position in the forehead. If a brow lift is planned, this anterior dissection is extended to the orbital rim where glabellar and sub-brow muscles can be transected, depending on the technique utilized.

To maximize scalp mobility, two to three complete coronal galeotomies are made, $15-20 \mathrm{~mm}$ apart. These galeotomies are carefully made just through the galea, careful to avoid transecting blood vessels immediately superficial to the galea that could compromise vascularity and healing. Several cycles of anterior traction are then applied to the frontal scalp through three towel clamps grabbing the leading edge, generating maximum creep. The frontal scalp is then held in its more anterior position, engaged onto two bioabsorbable fixation hooks that are typically used in endoscopic brow lifts (Endotine; Coapt, Palo Alto, California). These implants are anchored to the cranium through two holes drilled into the bone with a manual drill, each hole located just off the sagittal midline approximately $15 \mathrm{~mm}$ posterior to the anticipated location of the new hairline

With the scalp flap now secured into its anterior position, the redundant forehead skin can be excised, ideally along the previously marked out desired hairline position, and adjusted by several millimeters, depending on the actual location of the scalp flap. The forehead skin incision is performed with a matching bevel and contour to the initial tricophytic hairline incision to create some overlap by the forehead skin of the scalp containing partially transected follicles that will then grow hairs through the scar. Full-thickness forehead tissue, including skin, subcutaneous tissue and, frontalis muscle, is excised. This excision is best performed in a step-wise fashion, starting at the center where a deep suture is placed, then proceeding laterally $15 \mathrm{~mm}$ at a time in each direction placing another suture. The incision is closed using interrupted 2-0 Vicryl sutures for the deep layers (galea to frontalis muscle) at these $15 \mathrm{~mm}$ points, then a running 5-0 nylon for the skin edge, taking care to properly align the beveled skin edges of the tricophytic incision. Along the lateral extension of the incision into the temporal areas, any dog ear defect is worked out, and the incision is closed with interrupted 4-0 nylon sutures (Figure 4). Videos of the surgical procedure can be viewed on the following links (direct linkage from the online version of Forum):

Galeoetomies: http://youtu.be/9cORxblo3h0

First overlap: http://youtu.be/aYTLcJFkQZ8

Creep: http://youtu.be/1cilanNOrt8

Endotines: http://youtu.be/xzwmDtc36uM

Closure: http://youtu.be/ECQCPHwQiyo

Post-op patient: http://youtu.be/Zsw7DfSexbE 
Lowering the female hairline

$\checkmark$ from page 13

Antibiotic ointment is applied to the incision, and pressure dressing placed overnight. Hair washing can be resumed on day 4 , and sutures removed on days 6-8.

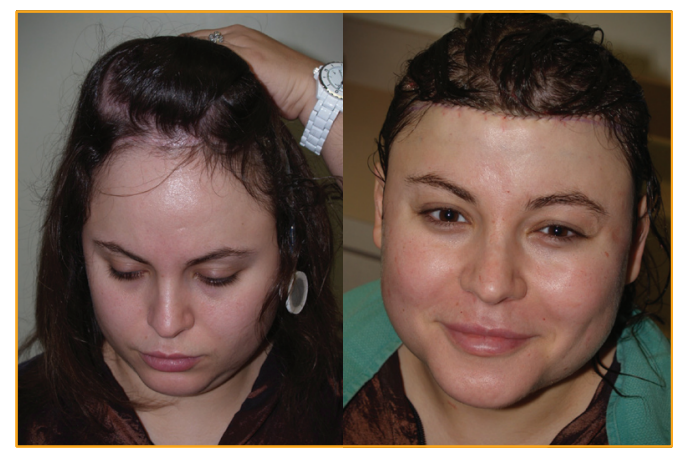

Figure 4. Before marked (left) and after (right) SHA.

\section{Optional: Tissue Expansion for Tight Scalps}

If scalp mobility is limited, a twostaged procedure utilizing tissue expansion can be performed. In the first stage, the expander is surgically placed under the frontal scalp that is to be expanded. Usually this, as well as the second stage procedure, is performed under oral sedation and local anesthesia. The expander, most commonly circular shaped 12 $15 \mathrm{~cm}$ in diameter, is placed subgaleal through a single hairline incision where the anticipated trichophytic hairline incision will be made with the second stage. With the balloon expander inserted into a relatively form-fitting subgaleal pocket, and the external injection port placed outside of the scalp with the tube exiting usually along the upper temporal region, the forehead incision is closed in layers (2-0 Prolene for the deeper tissues and 5-0 nylon for the skin edges). To take up the "dead space," $100-200 \mathrm{~mL}$ of saline is initially injected into the expander.

Approximately 2 weeks after expander insertion, twice weekly inflation begins. Using the sensation of significant tightness, pressure, or some pain as the indicator of maximum fill per expansion, anywhere from $30-100 \mathrm{~mL}$ of saline can be injected each time, for 8-12 weeks. Once sufficient expansion has been achieved, determined by the amount of excess tissue generated, the second stage is performed. This is essentially the same procedure as the non-expanded surgical hairline advancement as described above, with the expanded scalp advanced and excess forehead skin excised. Note that no galeotomies are indicated (Figures 5-7).

\section{Optional: Hair Grafting after SHA for Further Refinement}

Finally, hair grafting is sometimes a valuable adjunct to SHA, both to round

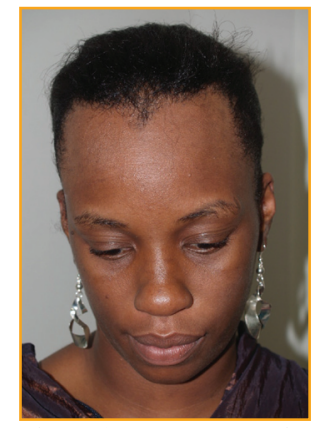

Figure 5. Before SHA, prior to the insertion of the balloon expander. The expansion was necessary due to the inadequate scalp laxity to achieve the desired 5-plus $\mathrm{cm}$ of hairline advancement.

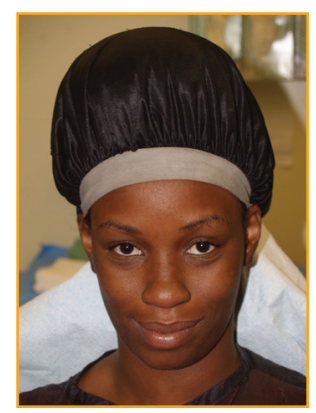

Figure 6. Patient with the balloon fully expanded, 8 weeks after its insertion, concealing the stretched out scalp with a loose head dressing. This photo was taken on the day the scalp was expanded.

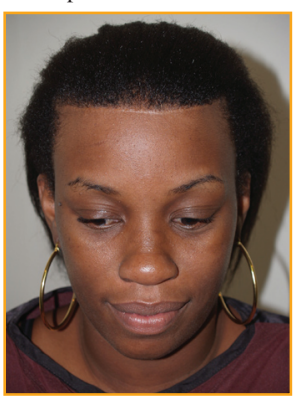

Figure 7. Patient 6 months after SHA, showing the much lower hairline achieved with tissue expansion in this highly motivated patient with tight scalp. out the hairline (filling in of the upper temporal and frontotemporal regions), as well as to conceal any hairline incision scar. It can also be utilized to create a widow's peak or other adjustment of design, and even to further advance the hairline that could not be achievable with the original hairline advancement procedure.

\section{Hairline Advancement Through Hair Transplantation}

Hair transplantation is a much more common way to advance the female hairline than SHA for a number of reasons. These include insufficient scalp laxity, ability to replace loss of the frontal and temporal hairs at the same time, a history of prior brow lift surgery, the desire to also round out the hairline and, very importantly, the procedure is not considered "surgery," and, thus, patient acceptance is usually higher.

Transplanting with follicular unit grafts provides the ideal combination of naturalness and, when placed closely together in recipient sites as small as $0.5 \mathrm{~mm}$, good to very good density. Each recipient site must be made at precisely the proper angles, which vary considerably in different areas of the hairline. In addition to placing the grafts in front of the existing hairline, the grafts can also be transplanted into areas of thinning farther back, helping to restore density. With hair transplantation, these grafts can be placed anywhere, allowing for excellent control in designing the shape of the hairline.

To be a good candidate, the patient should have adequate donor hair supply to achieve the desired amount of lowering of the hairline. Patients with a lot of miniaturization of the existing frontal hairline must be advised that there can be some shock loss to these hairs, resulting in a temporary higher appearing hairline until the transplanted hairs regrow.

\section{Surgical Technique of Hair Transplantation}

The areas to be transplanted, including the new frontal hairline, are marked out, and the hairs trimmed in the area of the donor strip. Similar to SHA, the procedure is done under local anesthesia with oral sedation. With the patient sitting upright, the donor strip is excised, then the incision closed with a 3-0 Prolene or, if dissolvable sutures are desired, a running 4-0 Caprosyn.

While the donor grafts are dissected under the microscope, the recipient sites are created. To ensure a proper and snug fit, test grafts are placed into recipient sites $0.5-0.8 \mathrm{~mm}$ in size, most commonly, with the 1-hair grafts usually placed into the 0.5 and sometimes $0.6 \mathrm{~mm}$ recipient sites, the 2-hair grafts into 0.6 or $0.7 \mathrm{~mm}$ sites, and the 3 - and 4-hair grafts into 0.7 or $0.8 \mathrm{~mm}$ sites. The angle and direction of the recipient sites are essentially made to mimic exactly those same attributes as the hairs in what is the existing hairline, just more anteriorly. This applies for all areas of the hairline-temporal and frontal-so that the effect is as if to move the entire existing hairline forward in the amount desired, which in the temporal region can range from several millimeters to as much as $3 \mathrm{~cm}$, while along the frontal region this can usually range between $1-3 \mathrm{~cm}$, but more if desired.

Along the frontal hairline, the objective is to create a natural irregularity, achieved with recipient sites made as a series of tiny anterior-projecting triangles three to four grafts deep, of closely packed 1-hair grafts in the first three rows, then 2-hair grafts in the center of each triangle as well as further back for another two to four rows most commonly, after which 3- and 4-hair grafts are placed to achieve density. Essentially, this results in the anteriormost $3-5 \mathrm{~mm}$ of the hairline having a soft, irregularly-irregular feathered composition to enhance its naturalness

While in general the angle of the recipient sites along the hair- 
line should be acutely anterior to avoid perpendicular orientation, which can look unnatural and of a lower density, mimicking the natural state of the direction of growth along the hairline often requires that the direction of hair growth will change all along the hairline. This means that certain areas of hair growth will be either to the right or left, then even reversing from an anterior to directly perpendicular, even posterior direction of growth. While all of these changes in direction, especially when in a posterior direction of growth, make it more difficult to achieve good density and complicate the process of graft insertion, it is essential to follow the existing hairline direction to create a truly natural appearing result and to avoid transecting hairs in the existing hairline higher up. Here is where only highly experienced and meticulous graft placers are essential. If a widow's peak is desired, it can usually be achieved with the placement of 10-20 hairs, usually not in a directly forward direction of growth, but rather usually off to one side or the other.

In the temporal region, the sites are made at a quite acute angle to the scalp, usually in a somewhat posterior as well as caudal direction. These recipient sites are made such that the frontal three to usually as many as six rows of grafts will contain 1 hair, then further back 2 hairs. Toward the more cephalic temporal area, the direction stays caudal but also goes from a posterior to more anterior direction, gradually blending into the frontotemporal recessions where the hairs typically grow more anteriorly.

Unlike the usually 90-minute-long SHA, these hair transplant procedures of 800 to as many as 2,800 follicular unit grafts can take as long as 8 hours to complete. Gentle hair washing is permitted on the second day, full activities on the sixth, and donor site sutures removed on the tenth day. Hair regrowth of the transplants usually begins at 4 months, with patients advised that impressive results are usually appreciated at 8-12 months. While most patients achieve adequate density with a single procedure, around $20 \%$ of patients-especially those with light colored scalps and dark hairs-will desire a second procedure of 300 to as many as 800 grafts, usually placed along the central area of the frontal hairline. This second procedure can be performed as soon as 10 months later (Figures 8 and 9).

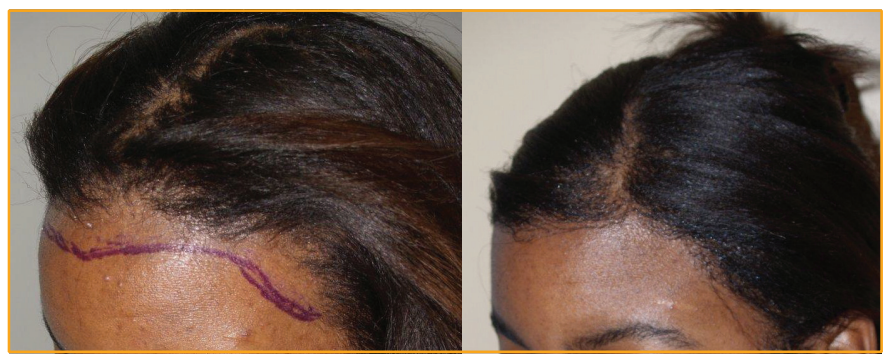

Figure 8. Before hair grafting $(l e f t)$ and after $($ right $)$ one procedure of 2,100 grafts to advance the hairline by transplantation.

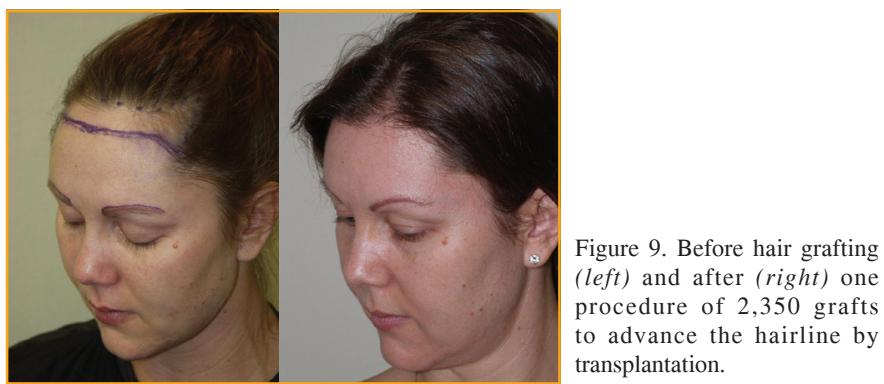

\section{Comment}

Both of these procedures are excellent when performed for the right patient and indication. While I recognize that not many hair restoration surgeons will feel comfortable performing SHA, it is important that a physician be able to identify those 5\%-10\% of patients who will benefit most from this procedure, and be ready to recommend it, and perhaps to learn how to perform it themselves. Satisfaction for my patients undergoing SHA is amongst the highest of any hair procedure, due to the rapidity of results, the reliability of hair growth, and the excellent hairline density, provided these patients realize the limitation in rounding of the hairline that will require future hair grafting.

Approximately one-third of SHAs have been performed on women of African ethnicity. This appears to be due to several causes, such as the high incidence of traction alopecia, the greater genetic propensity to be born with a high hairline, and lastly, the fact that many of these women seem to have quite elastic scalps, making possible $3 \mathrm{~cm}$ to as much as $5 \mathrm{~cm}$ of advancement without tissue expansion. Despite concerns of scarring, SHA (as well as hair grafting) procedures have not been characterized by this problem.

It is this risk of visible scarring along the hairline incision line that is perhaps the most significant concern regarding SHA for all patients considering it. In fact, this complication can be minimized with meticulous closure of the tricophytic incision, which will promote hair growth through the scar itself, thereby providing sufficient camouflage. Johnson and Cilento administered a patient-questionnaire-based review of more than 1,000 trichophytic or coronal approaches to the forehead. ${ }^{3}$ They found that greater than $98 \%$ of the patients were satisfied, would undergo the procedure again, and would recommend the procedure to others. Adjunctively, follicular unit grafting can be applied to visible scar regions for further camouflage as required.

Satisfaction with hair transplantation is quite high for most patients as well, provided they are advised of the possible need for a touch-up procedure to enhance density. The most common risk factor for a lower hair regrowth percentage is large procedures of over 2,200 or so grafts, which could be related to a compromise in blood flow (despite the making of very small recipient sites). The most challenging patients in which to achieve a good result with hair grafting are those with dark, coarse, straight hairs-something seen in higher incidence with those of Asian ethnicity. However, this challenge can be largely overcome with the transplanting of a large number of single-hair grafts deep into most of the frontal and temporal hairline, and careful angulation of recipient sites.

\section{References}

1. Beehner, M. Hairline design in hair replacement surgery. Facial Plast Surg. 2008; 24:389-403.

2. In: W.P. Unger, et al., eds. Hair Transplantation, 5th Ed. Chapter 18C. Informa HealthCare, London, UK, 2011; 496-503.

3. Cilento, B.W., and C.M.J. Johnson Jr. The case for open forehead rejuvenation: a review of 1004 procedures. Arch Facial Plast Surg. 2009; 11:13-17. 